\title{
Cardiovascular comorbidities associated with patients with chronic obstructive pulmonary disease - a hospital-based study
} Amit S. Gupta ${ }^{a}$, Venkitakrishnan Rajesh ${ }^{b}$, Ponneduthamkuzhy James ${ }^{c}$

\author{
Background Most patients with chronic obstructive \\ pulmonary disease (COPD) have cardiovascular \\ comorbidities (CVD), which significantly contribute to the \\ mortality and morbidity in these patients.
}

Objective The primary objective was to assess the prevalence of CVD in hospitalized patients with COPD. The secondary objectives were to study the risk factors associated with CVD in patients with COPD and to assess if the severity of COPD was related to the prevalence of CVD.

Patients and methods The present study was a prospective observational study. The study period spanned from January 2013 to December 2014. All consecutive patients admitted with a diagnosis of COPD were included in the study. Patients with coexisting chronic respiratory illnesses, like obstructive sleep apnea, interstitial lung disease, and bronchiectasis, were excluded. The presence of various CVD was assessed with a history, physical evaluation, and limited tests [ECG, troponin levels, serum brain natriuretic peptide (BNP), and echocardiography). $\chi^{2}$ test was used to test the statistical significance. Clearance from ethical committee and consent from patients were obtained before the study.

Results A total of 100 patients with COPD were included in the study. Overall, $60 \%$ of the patients had CVD. Common CVD were ischemic heart disease $(21 \%)$, congestive heart failure $(20 \%)$, stroke $(5 \%)$, and arrhythmias $(3 \%)$. The prevalence of CVD among patients with stages 1 and 2 COPD was $44.4 \%$, stage 3 COPD was $61.5 \%$ and stage 4 COPD

\section{Introduction}

A substantial proportion of patients with chronic obstructive pulmonary disease (COPD) have associated cardiovascular comorbidities (CVD), which in turn make significant contribution to morbidity and mortality in these patients. The prevalence of CVD varies between 30 and $70 \%$ in various studies. Epidemiological studies of cases and controls based on patient registries have demonstrated that patients with COPD have a greater percentage of chronic comorbidities, mainly cardiovascular [1-3]. It is well recognized that in patients with severe and very severe disease, the cause of death is determined by the underlying respiratory disease, which is in sharp contrast to patients with mild and moderate disease, where death owing to cancer and CVD is more frequent $[4,5]$. In recent years, a hypothesis has been generated that a systemic inflammatory process, present in patients with COPD, could be the link between this disease and extrapulmonary comorbidities.

There has been a paucity of data from the Indian subcontinent on the CVD in patients with COPD. was $66.7 \%(P=0.298)$. Using multiple linear regression analysis, significant risk factors for CVD were serum BNP levels $[P=0.001$; odds ratio $(\mathrm{OR}), 17.5]$, dyslipidemia $(P=0.037$; OR, 3.6), and systemic hypertension $(P=0.002$; OR, 4.6). Patients with COPD and CVD had a lower BMI as compared with those without CVD.

Conclusion Ischemic heart disease, congestive cardiac failure, and stroke were the most commonly identified CVD in patients with COPD. Systemic hypertension, dyslipidemia, and serum BNP levels were identified as important predictors for CVD in our study.

Egypt J Bronchol 2019 13:591-595

(C) 2020 Egyptian Journal of Bronchology

Egyptian Journal of Bronchology 2019 13:591-595

Keywords: cardiovascular comorbidity, chronic obstructive pulmonary disease, dyslipidemia, hypertension, serum brain natriuretic peptide

aDepartment of Pulmonary Medicine, Geetanjali Medical College and Hospital, Udaipur, Rajasthan, 'Department of Pulmonary Medicine, Rajagiri Hospital, 'Department of Pulmonary Medicine, Amrita Institute of Medical Sciences, Kochi, Kerala, India

Correspondence to Amit S. Gupta, DM, C 803, Archi Peace Park Apartment, Hiranmagri, Sector 4, Udaipur, Rajasthan, India. Postal Zip Code 313001. Tel: +91 953968 4584/91 875818 1614; e-mail: amitgupta86@yahoo.in

Received: 2 August 2019 Accepted: 1 October 2019 Published: 21 January 2020

The present study is therefore relevant and expects to throw some light on this hitherto under focused aspect of COPD care.

\section{Aims and objectives}

The primary objective of this study was to assess the prevalence of CVD in hospitalized patients with COPD.

The secondary objectives were to study the risk factors associated with the occurrence of CVD in patients with COPD and to assess if the severity of underlying COPD was related to the prevalence of CVD.

\section{Patients and methods}

The present study was a prospective observational study conducted at a tertiary care hospital in Kerala.

This is an open access journal, and articles are distributed under the terms of the Creative Commons Attribution-NonCommercial-ShareAlike 4.0 License, which allows others to remix, tweak, and build upon the work non-commercially, as long as appropriate credit is given and the new creations are licensed under the identical terms. 
The study period extended for 24 months from January 2013 to December 2014. All consecutive patients admitted to the pulmonary medicine unit during the study period were screened. Patients diagnosed to have COPD based on GOLD guidelines 2012 were included in the study [6]. Patients with coexisting chronic respiratory ailments other than COPD (like obstructive sleep apnea, interstitial lung disease, and bronchiectasis) were excluded from the study. Details of other coexisting comorbidities like systemic hypertension, diabetes mellitus, and dyslipidemia were recorded. History of previous CVD was taken, and all available past medical records were scrutinized. The presence of various CVD was assessed with a focused history, targeted physical evaluation, and limited battery of tests [12-lead ECG, troponin levels, serum brain natriuretic peptide (BNP), and $2 \mathrm{D}$ echocardiography]. If deemed necessary, formal evaluation by a cardiologist, coronary angiography, and right heart catheterization were undertaken on a caseto-case basis.

Percent prevalence rate of CVD (separately for each morbidity and overall) was computed. To test the statistical significance of the association of possible risk factors with CVD, $\chi^{2}$ test was used (SPSS, version 20). Multiple linear regression analysis was used to find the most significant risk factors associated with presence of CVD in patients with COPD.

Clearance from ethical committee and written consent from the study patients were obtained before the study.

\section{Results}

A total of 100 patients with COPD were included in the study. Many patients were in 60-80 (70\%) year age group, and most were males (87\%). The prevalence of CVD among patients with COPD in this study was $60 \%$. The mean age of study patients with and without CVD was similar (Table 1 and Fig. 1).

The most common CVD in our study was ischemic heart disease (21\%) followed by congestive heart failure (20\%) and stroke (5\%). The percent prevalence of each CVD is depicted in Fig. 2. The mean forced expiratory volume in $1 \mathrm{~s}$ (FEV1) value in patients with CVD was $40.09 \pm 14.42 \%$ of predicted normal, whereas in those without CVD was $46.09 \pm 17.61 \%$. This result was not statistically significant $(P=0.065)$. Overall, $74.5 \%$ of patients with systemic hypertension $(n=51 ; P=0.005)$ and $81.5 \%$ of patients with dyslipidemia $(n=27$; $P=0.015)$ had some CVD as compared with 44.9
Table 1 Baseline demographic characteristics

\begin{tabular}{lcr}
\hline \multicolumn{3}{c}{ Demographic characteristics } \\
\hline Age groups & $n(\%)$ & $P$ value \\
$41-50$ & $4(4)$ & \\
$51-60$ & $17(17)$ & \\
$61-70$ & $38(38)$ & \\
$71-80$ & $32(32)$ & \\
$81-90$ & $9(9)$ & \\
Total & $100(100)$ & \\
Mean age (years) & & \\
CVD & & \\
Present (60) & $68.40 \pm 8.65$ & \\
Absent (40) & $67.25 \pm 9.14$ & \\
\hline
\end{tabular}

CVD, cardiovascular comorbidities.

\section{Figure 1}

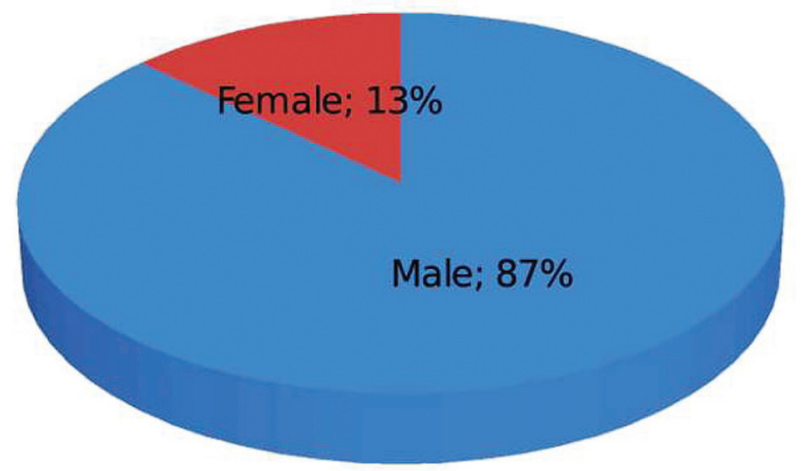

Sex distribution.

Figure 2

\section{No of patients}

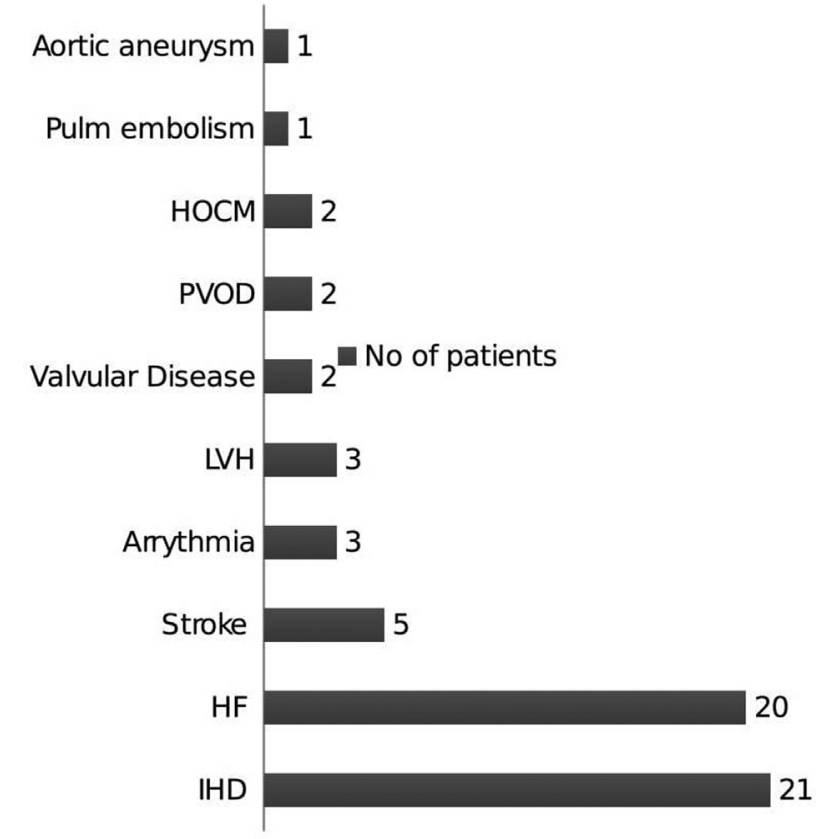

Prevalence of various cardiovascular comorbidities in patients with COPD. COPD, chronic obstructive pulmonary disease. 
and $52.1 \%$ of patients without systemic hypertension and dyslipidemia, respectively. This difference was of high statistical significance and indicated that patients with COPD with systemic hypertension and dyslipidemia had higher risk of CVD as compared with those without.

The prevalence of CVD among patients with stages 1 and 2 COPD was $44.4 \%$, stage 3 COPD was $61.5 \%$, and stage 4 COPD was $66.7 \%$. This difference did not reach statistical significance $(P=0.298)$, although a trend toward increasing CVD with increasing severity of COPD was observed (Table 2). Overall, 91.3\% of patients with elevated levels of BNP $(n=23)$ had some form of CVD as compared with $50.6 \%$ with normal levels of BNP. This difference was of high statistical significance $(P=0.001)$, indicating that patients with COPD with elevated levels of BNP have higher prevalence of CVD as compared with those with normal BNP levels. Using multiple linear regression analysis, it was found that systemic

Table 2 Relation between severity of chronic obstructive pulmonary disease and prevalence of cardiovascular comorbidities

\begin{tabular}{lcc}
\hline COPD stages & \% of patients with CVD & $P$ value \\
\hline Stages 1 and 2 (18) & 44.4 & 0.298 \\
Stage $3(52)$ & 61.5 & \\
Stage $4(30)$ & 66.7 & \\
\hline
\end{tabular}

COPD, chronic obstructive pulmonary disease; CVD, cardiovascular comorbidities. hypertension $(P=0.002)$, dyslipidemia $(P=0.037)$, and elevated serum BNP levels $(P=0.001)$ were significantly associated with presence of CVD in patients with COPD. The odd's ratio for presence of CVD was 4.6 for systemic hypertension, 3.6 for dyslipidemia, and 17.5 for elevated BNP levels. Elevated serum BNP levels were most significantly associated with the presence of CVD in our study as compared with all other risk factors (Fig. 3).

The mean BMI of patients with COPD with CVD was $25.85 \pm 4.19 \mathrm{~kg} / \mathrm{m}^{2}$, whereas for patients without CVD, the mean BMI was $27.46 \pm 3.98 \mathrm{~kg} / \mathrm{m}^{2}$. This result was not statistically significant $(P=0.057)$. The results tend to indicate that patients with COPD with CVD have a lower BMI as compared with those without CVD. Other factors that were found to be associated with increased risk of CVD but were not statistically significant were diabetes mellitus $(P=0.097)$, smoking status $(P=0.071)$, obesity BMI more than $30 \quad(P=0.239)$, increasing age $(P=0.526)$, and sex $(P=0.163)$.

\section{Discussion}

The present study showed a $60 \%$ prevalence of CVD in patients with COPD. The prevalence of CVD in general population in India was estimated to be $3-4 \%$ in rural areas and $8-10 \%$ in urban areas according to population-based cross-sectional surveys

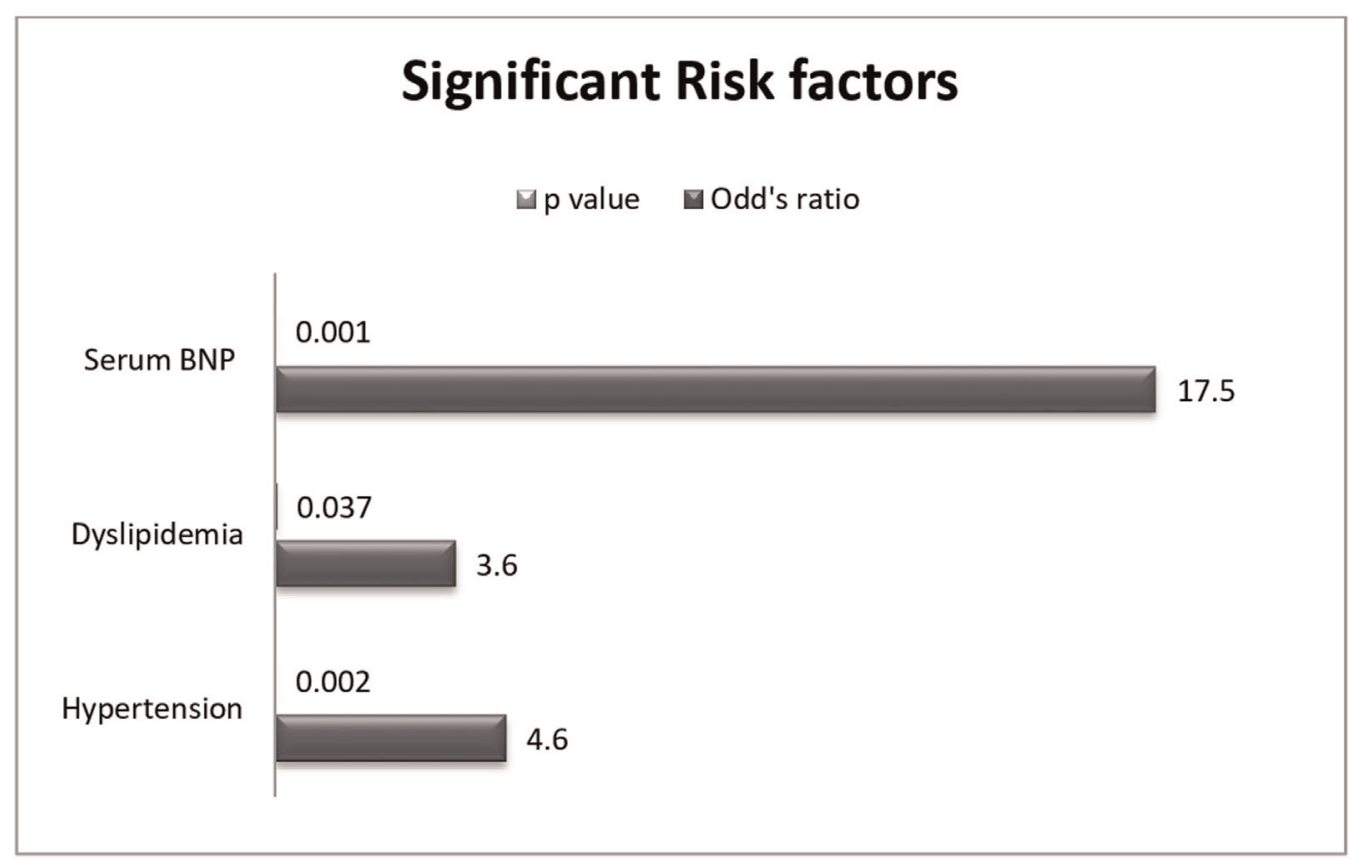

Significant risk factors for CVD in patients with COPD using multiple linear regression analysis. COPD, chronic obstructive pulmonary disease; CVD, cardiovascular comorbidities. 
in $2003[7,8]$. The overall prevalence of CVD in patients with COPD in various studies ranged from 28 to $70 \%[2,9,10]$. In the ECLIPSE (Evaluation of COPD Longitudinally to Identify Predictive Surrogate End-points) study, 'heart trouble' was reported in 26\% of 2164 patients with COPD, compared with $11 \%$ of 337 smoking controls, and myocardial infarction was reported in 9 versus 3\% [11]. Variations in study period, geographic peculiarities, and technique employed in each study to pick up CVD may account for the wide variations in prevalence observed across different studies.

It is now well established that reduced FEV1 is an independent risk factor for CVD independent of cigarette smoking [12]. In the present study, the prevalence of CVD among patients with stages 1 and 2 COPD was $44.4 \%$, stage 3 COPD was $61.5 \%$, and stage 4 COPD was $66.7 \%$. In congruence with existing literature, the mean FEV1 values in patients with CVD was lower (40.09 $\pm 14.42 \%)$ as compared with those without CVD $(46.09 \pm 17.61 \%)$ in our study. This indicates an increasing trend of CVD with falling FEV1 values and an inverse relationship between the two. This result was of borderline statistical significance $(P=0.065)$. Two previous studies have reported that the relative risk for CVD increased with increasing severity of airflow limitation $[12,13]$.

Malnutrition and cachexia are common among patients with COPD. The causes of cachexia in COPD are multifactorial which include increased work of breathing owing to abnormal respiratory mechanics, effect of several inflammatory cytokines, tissue hypoxia, and decreased oral intake [14]. In many cases, cachexia occurs despite adequate calorie intake, indicating that inflammatory catabolism plays a major role. In our study, the mean BMI for patients with CVD was $25.85 \pm 4.19 \mathrm{~kg} / \mathrm{m}^{2}$ whereas that for patients without CVD was $27.46 \pm 3.98 \mathrm{~kg} / \mathrm{m}^{2} \quad(P=0.057)$, suggesting that the prevalence of CVD is higher in patients with COPD with a lower BMI. Subgroup analysis revealed that patients with severe stages of COPD had a lower BMI, and hence the severity of COPD could be a confounding factor when assessing the effect of BMI. It may be reasonable to assume that the extrapulmonary spillover of systemic inflammation, which is more frequent is severe stages of COPD, plays a causative role in both CVD as well as cachexia.

Among all patients who had obesity, defined by BMI more than $30 \mathrm{~kg} / \mathrm{m}^{2}(n=25), 48 \%$ had some form of CVD as compared with $64 \%$ among those without obesity $(P=0.239)$. A retrospective observational study had demonstrated that middle-aged and older men with low body weight are at higher risk (relative risk, 2.76) of developing COPD even after adjusting for smoking history, age, and FEV1\% predicted [15]. Further 'obesity paradox' has been observed in patients with coronary heart disease and congestive heart failure. A systematic review of 40 cohort studies with 250152 patients found significantly lower risks for total mortality (relative risk, 0.87 ) and CVD (relative risk, 0.88) in overweight patients [16].

Finally, using multiple linear regression analysis, it was found that systemic hypertension $(P=0.002)$, dyslipidemia $(P=0.037)$, and elevated serum BNP levels $(P=0.001)$ were significantly associated with presence of CVD in patients with COPD. The odd's ratio for presence of CVD was 4.6 for systemic hypertension, 3.6 for dyslipidemia, and 17.5 for elevated BNP (Fig. 3). Systemic hypertension is reported in 40-60\% of patients with COPD [2,12]. In the ARIC and CHS population-based cohorts, the prevalence of hypertension was $34 \%$ in normal participants but $40 \%$ in GOLD stage 1 patients with COPD, $44 \%$ in GOLD stage 2, and $51 \%$ in GOLD stages 3 and 4 patients with COPD, with an odd's ratio of having hypertension of 1.4 in GOLD stage 2 and 1.6 in GOLD stages 3 and 4 compared with normal participants [12]. The reported prevalence of dyslipidemia in COPD in various studies is $9-51 \%$ $[17,18]$. Serum BNP is an established marker of ventricular dysfunction and is often elevated in patients with acute exacerbation of COPD. It indicates poor outcome with increased 30-day mortality. In a study conducted by Chang et al. [19], of 250 patients of COPD admitted with exacerbation, 65 (26\%) patients had elevated serum BNP levels. Similarly in a study conducted by McCullogh et al. [20], a cutoff point of $100 \mathrm{pg} / \mathrm{ml}$ had a sensitivity of $93.1 \%$ and specificity of $77.3 \%$, accuracy of $80.6 \%$ for predicting presence of congestive cardiac failure. Elevated BNP levels were most significantly associated with the presence of CVD in our study, as compared with all other risk factors.

\section{Limitations of the study}

The present study was a hospital-based study conducted in patients with COPD admitted during exacerbations. The systemic inflammation tends to worsen during acute exacerbations and consequently, cardiac events tend to increase. Outpatients with COPD, who form the huge bulk, could not be targeted because of the study design. Invasive tests like coronary angiography was not employed to 
reveal occult coronary artery disease in the study patients, and the study might have underestimated frequency of ischemic heart disease. The risk factors identified for the risk for CVD in patients with COPD are well-known cardiac risk factors even in the absence of lung disease, and they may be confounding factors.

\section{Conclusion}

The prevalence of CVD among patients with COPD in our study was found to be $60 \%$, with most common CVD in our study being ischemic heart disease, congestive heart failure, and stroke. Using multiple linear regression analysis, it was found that systemic hypertension, dyslipidemia, and elevated serum BNP levels were significantly associated with presence of CVD in patients with COPD.

\section{Financial support and sponsorship \\ Nil.}

\section{Conflicts of interest}

There are no conflicts of interest.

\section{References}

1 Mannino DM, Buist AS, Petty TL, Enright PL, Redd SC. Lung function and mortality in the United States: data from the First National Health and Nutrition Examination Survey follow up study. Thorax 2003; 58:388-393.

2 Curkendall S, DeLuise C, Jones JK, Lanes S, Stang MR, Goehring E, et al. Cardiovascular disease in patients with chronic obstructive pulmonary disease, Saskatchewan Canada cardiovascular disease in COPD patients. Ann Epidemiol 2006; 16:63-70.

3 Huiart L, Ernst P, Suissa S. Cardiovascular morbidity and mortality in COPD. Chest 2005; 128:2640-2646.

4 Hansell AL, Walk JA, Soriano JB. What do chronic obstructive pulmonary disease patients die from? A multiple cause coding analysis. Eur Respir J 2003; 22:809-814.
5 Mannino DM, Doherty DE, Buist AS. Global Initiative on Obstructive Lung Disease (GOLD) classification of lung disease and mortality: findings from the Atherosclerosis Risk in Communities (ARIC) study. Respir Med 2006; 100:115-122.

6 Global Initiative for Chronic Obstructive Lung Disease (GOLD). Global Strategy for the Diagnosis, Management and Prevention of COPD. 2012. Available from: http://goldcopd.org

7 Gupta R. Burden of coronary heart disease in India. Indian Heart J 2005; 57:632-638.

8 Gupta R. Coronary heart disease in India: absolute numbers and economic burden.Rapid response to Ghaffar A, Reddy KS, Singhi M. Burden of noncommunicable diseases in South Asia. Br Med J 2004; 328:807-810.

9 Feary JR, Rodrigues LC, Smith CJ, Hubbard RB, Gibson JE. Prevalence of major comorbidities in subjects with COPD and incidence of myocardial infarction and stroke: a comprehensive analysis using data from primary care. Thorax 2010; 65:956-962.

10 Finkelstein J, Cha E, Scharf SM. Chronic obstructive pulmonary disease as an independent risk factor for cardiovascular morbidity. Int $J$ Chron Obstruct Pulmon Dis 2009; 4:337-349.

11 Agusti A, Calverley PMA, Celli B, Coxson HO, Edwards LD, Lomas DA, et al. Characterisation of COPD heterogeneity in the ECLIPSE cohort. Respir Res 2010; 11:122.

12 Mannino DM, Thorn D, Swensen A, Holguin F. Prevalence and outcomes of diabetes, hypertension and cardiovascular disease in COPD. Eur Respir J 2008; 32:962-969.

13 Johnston AK, Mannino DM, Hagan GW, Davis KJ, Kiri VA. Relationship between lung function impairment and incidence or recurrence of cardiovascular events in a middle-aged cohort. Thorax 2008; 63:599-605.

14 King DA, Cordova F, Scharf SM. Nutritional aspects of chronic obstructive pulmonary disease. Proc Am Thorac Soc 2008; 5:519-523.

15 Harik-Khan RI, Fleg JL, Wise RA. Body mass index and the risk of COPD. Chest 2002; 121:370-376.

16 Romero-Corral A, Montori VM, Somers VK, Korinek J, Thomas RJ, Allison $\mathrm{TG}$, et al. Association of bodyweight with total mortality and with cardiovascular events in coronary artery disease: a systematic review of cohort studies. Lancet 2006; 368:666-678.

17 Lusis AJ. Atherosclerosis. Nature 2000; 407:233-241

18 Verma S, LiSH, Badiwala MV, Weisel RD, Fedak PW, Li RK, et al. Endothelin antagonism and interleukin 6 inhibition attenuate the proatherogenic effects of C-reactive protein. Circulation 2002; 105:1890-1896.

19 Chang CL, Robinson SC, Mills GD, Sullivan GD, Karalus NC, McLachlan JD, Hancox RJ. Biochemical markers of cardiac dysfunction predict mortality in acute exacerbation of COPD. Thorax 2011; 66:764-768.

20 McCullogh PA, Hollander JE, Nowak RM, Storrow AB, Duc P, Omland T, et al. Uncovering heart failure in patients with a history of pulmonary disease: rationale for the early use of B-type Natriuretic peptide in the emergency department. Acad Emerg Med 2003; 10:198-204. 